\title{
UNIVERSALITY OF SMALL LATTICE VARIETIES
}

\author{
V. KOUBEK AND J. SICHLER ${ }^{1}$
}

\begin{abstract}
There exists a finitely generated lattice variety $S$ such that the class of all nonconstant homomorphisms between members of $S$ contains a universal category as a full subcategory. In particular, every monoid $M$ is isomorphic to the monoid of all nonconstant endomorphisms of a lattice from $S$, and $S$ contains arbitrarily large lattices representing $M$. The category of all $(0,1)$-homomorphisms of lattices in $S$ is also shown to be universal.
\end{abstract}

1. Introduction. The well-known representation of an arbitrary group as the automorphism group of a distributive lattice obtained by Garret Birkhoff [4] is one of the early results on lattice varieties viewed as categories. Since distributive lattices form the smallest nontrivial lattice variety, Birkhoff's result points to richness of the homomorphism structure of small lattice varieties. The distributive lattices themselves, however, are determined up to anti-isomorphism by their endomorphism monoids (see B. M. Schein [11]), and distributive (0,1)-lattices are similarly determined by their $(0,1)$-endomorphisms (R. McKenzie and C. Tsinakis $[\mathbf{9}])$. These monoids always contain left zeros and thus the Birkhoff representation cannot be extended to monoids of $(0,1)$-endomorphisms of distributive lattices.

The variety of all $(0,1)$-lattices and their $(0,1)$-homorphisms, on the other hand, is universal [5] and, consequently, every monoid occurs as the monoid of all $(0,1)$ endomorphisms of some lattice. Recall that a category $\mathbf{C}$ is universal (or binding) if every category of algebras and all their homomorphisms are isomorphic to a full subcategory of $\mathbf{C}$. Any universal category contains a proper class of pairwise nonisomorphic objects with no nontrivial endomorphisms (called rigid objects) [10]; the category of all lattice homomorphisms fails to be universal since every constant mapping is a lattice homomorphism, so that all rigid lattices are trivial.

Nonconstant lattice homomorphisms, on the other hand, form a universal category $[\mathbf{1 0}, \mathbf{1 2}]$; that is, all lattices form an almost universal category.

These facts suggest investigations of proper $(0,1)$-lattice varieties that are universal or lattice varieties that are almost universal. A universal locally finite $(0,1)$ lattice variety $A$ was described in $[\mathbf{3}]$ and, subsequently, two of its proper subvarieties were shown to be $(0,1)$-universal $[\mathbf{7}]$. Here we exhibit finitely generated (i.e., generated by a finite lattice) almost universal subvarieties of $A$. This also answers a question by Ervin Fried concerning the existence of a finitely generated congruence distributive universal variety of algebras; in this connection, see also [8].

THEOREM 1.1. There are infinitely many almost universal lattice varieties generated by a finite lattice.

Received by the editors March 9, 1983 and, in revised form, August 25, 1983.

1980 Mathematics Subject Classification. Primary 06B20, 08A35; Secondary 18B15.

${ }^{1}$ The support of the NSERC is gratefully acknowledged by the authors. 
THEOREM 1.2. There are infinitely many universal varieties of $(0,1)$-lattices generated by a finite $(0,1)$-lattice.

All these finitely generated varieties are contained in $A$. Our method of proof is based on a somewhat unexpected result of $[\mathbf{1}]$ showing that homomorphisms of distributive lattices preserving finitely many constants form a universal category.

2. The construction. Any pair $G=(V, E)$ in which $E$ is a set of two-element subsets (called edges) of the vertex set $V$ is an undirected graph. An isolated vertex of a graph $G$ is contained in none of its edges. A mapping $f: V \rightarrow V^{\prime}$ is compatible if $\{f(v), f(w)\} \in E^{\prime}$ for every $\{v, w\} \in E$. A finite $A \subseteq V$ is an independent set of $G$ if $\{v, w\} \subseteq A$ for no edge $\{v, w\}$ of $G$. Finally, $N(v)=\{w \in V:\{v, w\} \in E\}$ will be called the neighbourhood of a vertex $v$ in $G$.

Add a largest element 1 to the inclusion ordered poset of all independent sets of $G$; the resulting poset is a lattice called the lower cover set lattice $I_{*}(G)$ associated with $G$. For $a, b \in I_{*}(G) \backslash\{1\}$, the meet is defined by $a \wedge b=a \cap b$, while $a \vee b=1$ if $a \cup b$ is dependent and $a \vee b=a \cup b$ otherwise. The empty set is the zero of this lattice, and its atoms are exactly all singletons $\{v\}$ for $v \in V$. Note that every proper ideal of $I_{*}(G)$ is a distributive lattice.

Let $G=(V, E)$ be a finite graph and $z \in V$; set $W=V \backslash\{z\}$ and let $H$ denote the subgraph of $G$ induced by $W$. Since every independent set of $H$ is also an independent set of $G$, the cover set lattice $I_{*}(H)$ is isomorphic to a $(0,1)$-sublattice of $I_{*}(G)$. Assume $J \subseteq W$ to be disjoint with $N(z)$.

Given a distributive lattice $D$ with a zero $z$, let $D^{+}$denote the lattice obtained by adding a new zero 0 and a new unit 1 to $D$. Clearly, $D^{+}$is a distributive $(0,1)$ lattice; for every lattice homomorphism $f: D \rightarrow D^{\prime}$, the mapping $f^{+}$extending $f$ by $f^{+}(0)=0, f^{+}(1)=1$ is a $(0,1)$-homomorphism of $D^{+}$into $\left(D^{\prime}\right)^{+}$.

Let $P=(P(j): j \in J)$ be a system of proper nonvoid prime filters of the original distributive lattice $D$. In the cartesian product $D^{+} \times I_{*}(H)$ let $R=R(P)$ be the order filer generated by

$$
\bigcup(P(j) \times\{\{j\}\}: j \in J) \cup\{(z,\{v\}): v \in N(z)\} \cup\{(1,0),(0,1)\},
$$

and let $\Gamma\left(D^{+}, I_{*}(H) ; R\right)=\left(\left(D^{+} \times I_{*}(H)\right) \backslash R\right) \cup\{1\}$, where 1 is the largest element of $\Gamma\left(D^{+}, I_{*}(H) ; R\right)$. The resulting poset clearly is a $(0,1)$-lattice, not containing any elements of the form $(1, q),(d, 1)$, whose proper ideals are all distributive. Its atoms are $(z, 0)$ and all the elements $(0,\{w\})$ for $w \in W$. The following claim is obvious.

LEMmA 2.1. The lattice $\Gamma=\Gamma\left(D^{+}, I_{*}(H) ; R\right)$ contains both $D^{+}$and $I_{*}(H)$ as $(0,1)$-sublattices. The set $A=\{(z, 0)\} \cup\{(0,\{w\}): w \in W\}$ of all atoms of $\Gamma$ generates a $(0,1)$-sublattice isomorphic to $I_{*}(G)$.

LEMMA 2.2. If $d_{0}, d_{1} \in D,\left(d_{i}, q_{i}\right)<1$ in $\Gamma$ for $i=0,1$, and $q_{0} \cup q_{1}$ is an independent set of $H$, then $\left(d_{0}, q_{0}\right) \vee\left(d_{1}, q_{1}\right)=1$ if and only if there are $i \in\{0,1\}$ and $j \in J$ such that $j \in q_{1-i} \backslash q_{i}$ and $d_{i} \in P(j), d_{1-i} \notin P(j)$.

PROOF. Since $d_{0} \vee d_{1}<1$ and $q_{0} \vee q_{1}<1$ in the respective sublattices of $\Gamma$, by (1) there are two cases in which $\left(d_{0} \vee d_{1}, q_{0} \vee q_{1}\right)=\left(d_{0} \vee d_{1}, 0\right) \vee\left(0, q_{0} \vee q_{1}\right)=1$ : either $\left(q_{0} \cup q_{1}\right) \cap N(z) \neq \emptyset$, so that, contrary to the hypothesis, $\left(d_{i}, q_{i}\right) \in R(P)$ for 
at least one of these elements; or else there is a $j \in J$ such that $d_{0} \vee d_{1} \in P(j)$ and $j \in q_{0} \cup q_{1}$. Since $P(j)$ is a prime filter, $d_{i} \in P(j)$ for some $i$ and $j \in q_{1-i} \backslash q_{i}$, for otherwise $\left(d_{i}, q_{i}\right) \in R(P)$. Should $d_{1-i} \in P(j)$ as well, $\left(d_{1-i}, q_{1-i}\right) \in R(P)$. This contradiction concludes the proof for the converse implication is trivial.

LEMMA 2.3. If $A, A^{\prime}, B, B^{\prime}$ are $(0,1)$-lattices and $R \subseteq A \times B, R^{\prime} \subseteq A^{\prime} \times B^{\prime}$ are order filters, then, for every $(0,1)$-homomorphism $g: A \times B \rightarrow A \times B$ satisfying

$$
g(a, b) \in R^{\prime} \quad \text { if and only if }(a, b) \in R,
$$

the mapping $g^{\#}: \Gamma(A, B ; R) \rightarrow \Gamma\left(A^{\prime}, B^{\prime} ; R^{\prime}\right)$ defined as the extension of $g \uparrow$ $((A \times B) \backslash R)$ by $g^{\#}(1)=1$ is a $(0,1)$-homomorphism such that $g^{\#}(y)=1$ only if $y=1$.

PROOF. Since 1 is the largest element of both lattices, the mapping $g^{\#}$ is meetpreserving. The rest follows by (2).

Notation. For any finite set $J$, let $\mathbf{D}(J)$ denote the category of all pairs $(D,(P(j)$ : $j \in J))^{\circ}$ such that $D$ is a distributive lattice with a zero $z$ and $P(j)$ is a proper nonvoid prime filter of $D$ for every $j \in J$. A lattice homomorphism $f: D \rightarrow D^{\prime}$ is a morphism of $\mathbf{D}(J)$ from $(D,(P(j): j \in J))$ to $\left(D^{\prime},\left(P^{\prime}(j): j \in J\right)\right)$ whenever, for every $j \in J$,

$$
f(d) \in P^{\prime}(j) \quad \text { if and only if } d \in P(j) .
$$

The following result of $[\mathbf{1}]$ is crucial.

THEOREM $2.4[\mathbf{1}]$. There is a nonempty finite set $J$ such that the category $\mathbf{D}(J)$ is universal. Furthermore, there exists a full embedding $\Psi: \mathbf{G} \rightarrow \mathbf{D}(J)$ of the category $\mathbf{G}$ of all graphs and all their compatible mappings such that $\Psi(G)$ is finite for every finite graph $G$. Consequently, every finite monoid occurs as the endomorphism monoid of infinitely many nonisomorphic finite objects in $\mathbf{D}(J)$.

Let $G=(V, E)$ and $H$ be graphs as above. Set

$$
\Phi(D,(P(j): j \in J))=\Gamma\left(D^{+}, I_{*}(H) ; R(P)\right)
$$

for every object $(D, P)=(D,(P(j): j \in J))$ of $\mathbf{D}(J)$, with $R=R(P)$ defined by (1). Furthermore, for every morphism $f$ of $\mathbf{D}(J)$ define $\Phi(f): \Phi(D, P) \rightarrow \Phi\left(D^{\prime}, P^{\prime}\right)$ by $\Phi(f)=\left(f^{+} \times \mathrm{id}\right)^{\#}$; it is easily verified that $(a, q) \in R$ if and only if $\left(f^{+}(a), q\right) \in R^{\prime}$. From 2.2 and 2.3 it follows that $\Phi(f)$ is a $(0,1)$-homomorphism separating 1 ; since $\Phi(f)(d, q)=\left(f^{+}(d), q\right)$ for all $(d, q) \in \Phi(D, P) \backslash\{1\}, \Phi$ is a faithful functor from $\mathbf{D}(J)$ into the category of all $(0,1)$-preserving lattice homomorphisms. To prove Theorems 1.1 and 1.2 we need to select a graph $G$ in such a way that $\Phi_{G}$ is a full functor. First of all, however, we show that the image of $\Phi_{G}$ is contained in a finitely generated variety.

For a finite nonempty set $J$, let $T$ be the boolean lattice of all subsets of the $(|J|+$ 1)-element set $\{a(j): j \in J\} \cup\{e\}$, and let $z$ be the zero of $T$. If $F(j)=[\{a(j)\})$ is the principal filter of $T$ generated by $\{a(j)\}$, then $(T,(F(j): j \in J)) \in \mathbf{D}(J)$ since $\{a(j)\}$ is join irreducible. For every homomorphism $h: D \rightarrow\{z,\{e\}\}$ of a distributive lattice $D$ onto the two-element chain with $z<\{e\}$ and for any system $P=(P(j): j \in J)$ of prime filters in $D$ there is a morphism $h_{1}:(D, P) \rightarrow(T,(F(j): j \in J))$ of $\mathbf{D}(J)$ with $\operatorname{Ker}\left(h_{1}\right) \subseteq \operatorname{Ker}(h)$, namely the mapping defined by $h_{1}(d)=h(d) \cup\{a(j): d \in P(j)\}$. 
Hence $\Phi\left(h_{1}\right)$ is a $(0,1)$-homomorphism of $\Phi(D, P)$ into $\Phi(T,(F(j): j \in J))$ such that $\Phi\left(h_{1}\right)^{-1}\{1\}=\{1\}$. To show that $\Phi(D, P)$ is a sublattice of some power of $\Phi(T,(F(j): j \in J))$ it is thus enough to consider elements $(a, p)$ of $\Phi(D, P) \backslash\{1\}$. For any $h: D \rightarrow\{z,\{e\}\}$ we obtain $\Phi\left(h_{1}\right)(a, p)=\left(\left(h_{1}\right)^{\dagger}(a), p\right)$; since $\left(h_{1}\right)^{+}$separates 0 and 1 , and because each pair of elements of $D$ is separated by some $h$ whose kernel contains that of $h_{1}$, we find that $\Phi(D, P)$ indeed lies in the variety generated by the finite lattice $\Phi(T,(F(j): j \in J))$.

It is easily seen that $\Phi(T,(F(j): j \in J))$ is isomorphic to a $(0,1)$-sublattice of the lower cover set lattice $I_{*}(V \cup\{a(j): j \in J\} \cup\{e\}, F)$, where $F=E \cup\{\{a(j), j\}: j \in$ $J\}$ : the mapping defined by $k(1)=1$ and $k(b, q)=b \cup q$ for $(b, q)<1$ is the desired embedding. Thus $\Phi$ is a faithful functor that maps $\mathbf{D}(J)$ into a variety generated by a single finite cover set lattice; any such variety is contained in the variety $A_{*}$ generated by all lower cover set lattices associated with undirected graphs. The variety $A$ is the join of $A_{*}$ and of the variety $A^{*}$ formed by duals of lattices from $A_{*}$. This proves the claim below.

PROPOSITION 2.5. For every finite graph $G$ containing $J$ in its vertex set there is a variety $S \subset A_{*}$ generated by a finite cover set lattice and a faithful functor $\Phi_{r}=\Phi$ of $\mathbf{D}(J)$ into the class of all $(0,1)$-homomorphisms of lattices in $S . A$ similar statement holds for $A^{*}$.

Recall that $W=V \backslash\{z\}$ is contained in the vertex set of the graph $G$ used to define $\Phi=\Phi_{G}$. The following lemma is needed to prove that $\Phi$ is a full functor.

LEMMA 2.6. If $h: \Phi(D, P) \rightarrow \Phi\left(D^{\prime}, P^{\prime}\right)$ is a $(0,1)$-homomorphism separating i such that $h_{\iota}(z, 0) \geq(z, 0)$ and $h(0,\{w\})=(0,\{w\})$ for all $w \in W$ then there is a unique morphism $f:(D, P) \rightarrow\left(D^{\prime}, P^{\prime}\right)$ with $\Phi(f)=h$.

PRoOF. If $d \in D$ then

$$
h(d, 0) \wedge(0,\{w\})=h((d, 0) \wedge(0,\{w\}))=h(0,0)=(0,0)
$$

fur every $w \in W$, so that $h(d, 0)=(a, 0)<1$; simultaneously, $(z, 0) \leq h(z, 0) \leq$ $h^{\prime}(d, 0)=(a, 0)$ and $a \in D^{\prime}$ follows. The mapping $f$ defined by $(f(d), 0)=h(d, 0)$ is thus a lattice homomorphism of $D$ into $D^{\prime}$. Since $h$ preserves and separates $1,(d, 0) \vee(0,\{j\})=1$ if and only if $(f(d), 0) \vee(0,\{j\})=1$. Lemma 2.2 applied to these equalities shows that $d \in P(j)$ is equivalent to $f(d) \in P^{\prime}(J)$ for every $j \in J$; hence $f$ is a morphism in $\mathbf{D}(J)$. From $(0, q)=\bigvee((0,\{w\}): w \in q)$ it follows that $h(0, q)=(0, q)$ whenever $(0, q)<1$; whence $h(d, q)=h((d, 0) \vee(0, q))=(f(d), q)$, aind $h=\left(f^{+} \times \mathrm{id}\right)^{\#}=\Phi(f)$ as was to be shown.

By $[6$ or 10], there exist arbitrarily large finite automorphism-free graphs satisfying $1 \leq N(w) \leq 4$ for all vertices $w$. Let $H=(W, F)$ be such a graph with $|W| \geq$ $|J|+\max (|J|, 6)$, where $J$ is the finite set from Theorem 2.4. Assume $J \subseteq W \not \supset z$, and let $G=(V, E)$ be the graph with $V=W \cup\{z\}, E=F \cup\{\{z, w\}: w \in W \backslash J\}$. Since $z$ is the only vertex of $G$ with $|N(z)|>5$, the graph $G$ is automorphism-free; it is easily seen that $G$ has no isolated vertices and that $H$ is an induced subgraph of $G$. Furthermore, $G$ satisfies

$$
V=N(z) \cup J \quad \text { and } \quad N(z) \cap J=\emptyset,
$$

$$
2|J|<|V|,
$$




$$
|N(w)|<|N(z)|-1 \quad \text { for all } w \in V \backslash\{z\} .
$$

LEMMA $2.7[\mathbf{3}]$. If $G$ is an automorphism-free graph without isolated vertices then $I_{*}(G)$ is a simple lattice.

From Lemma 2.1 we recall that the set $A=\{(z, 0)\} \cup\{(0,\{w\}): w \in W\}$ of atoms of any $\Phi_{G}(D, P)$ generates the $(0,1)$-sublattice $I_{*}(G)$. For every $y \in \Phi_{G}(D, P)$ let $A(y)$ denote the set of atoms below $y$ in $\Phi_{G}(D, P)$; clearly $A(x \wedge y)=A(x) \cap A(y)$ holds for all $x, y \in \Phi(D, P)$.

PROPOSITION 2.8. If $G$ is an automorphism-free finite graph with no isolated vertices satisfying (4), (5), and (6), then every nonconstant homomorphism $h: \Phi_{G}(D, P) \rightarrow \Phi_{G}\left(D^{\prime}, P^{\prime}\right)$ is of the form $h=\Phi_{G}(f)$ for some $f:(D, P) \rightarrow\left(D^{\prime}, P^{\prime}\right)$.

ProOF. Assume $h$ to be nonconstant; by Lemma 2.7 the restriction of $h$ to $I_{*}(G)$ must be one-to-one. Since every proper ideal of $\Phi_{G}\left(D^{\prime}, P^{\prime}\right)$ is distributive and $I_{*}(G)$ has more than two elements, $h(1)=1$ follows. For every $(b, q)<1$ there is an atom $a$ with $a \wedge(b, q)=(0,0)$, for otherwise all vertices of $G$ would have to be isolated. Thus $h(b, q)=1$ yields contradictory $h(a)=h(0,0)$. It follows that $h$ preserves and separates the unit 1 .

Denote $h(0,0)=(d, q)$, and $h(a)=(d(a), q(a))$ for every $a \in A$. Let $\alpha(x)=$ $A(h(x))$ for every $x \in \Phi_{G}(D, P)$.

Assume $d>0$ first. Thus, for every $a \in A, d(a) \geq d \geq z$, and $q(a) \subseteq J$ follows from $h(a)<1$ by (4). Let $B=\{b \in A: q(b)>q\}$; for every $b \in B$ there exists some

$$
g(b) \in q(b) \backslash \bigcup\left(q\left(b^{\prime}\right): b^{\prime} \in B \backslash\{b\}\right) \subseteq J,
$$

so that $|B| \leq|J|$. Since $G$ has no isolated points, for every $c \in A \backslash B$ there exists $a \in A$ such that

$$
(d(c), q) \vee(d(a), q(a))=h(c) \vee h(a)=h(c \vee a)=h(1)=1 .
$$

From $q \subseteq q(a)$ and Lemma 2.2 it follows that $d(c) \in P(j)$ for some $j \in q(a) \backslash q \subseteq J$. If $c^{\prime} \in A \backslash B$ is distinct from $c$ and $d\left(c^{\prime}\right) \in P(j)$ then $d=d(c) \wedge d\left(c^{\prime}\right) \in P(j)$, and hence

$$
1 \leq(d, 0) \vee(0, q(a)) \leq(d(a), 0) \vee(0, q(a)),
$$

which contradicts $h(a)<1$. Therefore $|A \backslash B| \leq|J|$; consequently, $|V|=|A| \leq 2|J|$ contrary to $(5)$.

As a result, $h(0,0)=(0, q)$ for some $q \subseteq W$ and, in particular, $h(0,0)$ is the join of $\alpha(0,0)$. If $\alpha(a) \subseteq \bigcup(\alpha(b): b \in A \backslash\{a\})$ then $\alpha(a)=\alpha(0,0)$, and $h(a)=h(0,0)$ follows. Hence $\alpha(a) \backslash \alpha(0,0)$ is nonempty for every $a \in A$; the finiteness of $A$ yields $h(0,0)=(0,0)$ as well as the existence of a permutation $p: A \rightarrow A$ such that $\alpha(a)=\{p(a)\}$ on $A$. Furthermore, $h(a)=p(a)$ whenever $p(a) \neq(z, 0)$. If $p(z, 0)=(0,\{w\})$ for some $w \in W$, then for every $v \in N(z)$ with $p(0,\{v\}) \neq(z, 0)$ we obtain

$$
p(0,\{v\}) \vee(0,\{w\})=h(0,\{v\}) \vee h(z, 0)=1,
$$

in contradiction to $(6)$. Hence $h(z, 0) \geq p(z, 0)=(z, 0)$ and $h(0,\{w\})=p(0,\{w\})$ for all $w \in W$. It is now routine to verify that $p$ defines an automorphism of the induced subgraph $H$ of $G$; since $H$ has no nontrivial automorphisms, $p(0,\{w\})=$ $(0,\{w\})$ for all $w \in W$. Lemma 2.6 now applies to conclude the proof. 
Since there are infinitely many finite subdirectly irreducible lower cover set lattices $[\mathbf{3}]$, Jónsson's Lemma shows that infinitely many finitely generated universal lattice varieties of either type exist already in $A_{*}$. Every finitely generated subvariety of $A_{*}$ is thus contained in a finitely generated variety that is universal in either sense.

Since every full embedding $\Phi_{G}$ preserves finiteness, Theorem 2.4 implies that every finite monoid is isomorphic to the $(0,1)$-endomorphism monoid of infinitely many finite nonisomorphic members of each of the constructed finitely generated varieties; this observation sharply contrasts with $[\mathbf{9}, \mathbf{1 1}]$, and considerably improves the main representation result of $[\mathbf{2}]$.

Problem. Characterize minimal finitely generated $(0,1)$-universal or almost universal subvarieties of $A_{*}$.

\section{REFERENCES}

1. M. E. Adams, V. Koubek and J. Sichler, Homomorphisms of distributive lattices, Houston J. Math. (to appear).

2. M. E. Adams and J. Sichler, Bounded endomorphisms of lattices of finite height, Canad. J. Math. 29 (1977), 1254-1263.

3. _ Cover set lattices, Canad. J. Math. 32 (1980), 1177-1205.

4. G. Birkhoff, On groups of automorphisms, Rev. Un. Mat. Argentina 11 (1946), 155-157.

5. G. Grätzer and J. Sichler, On the endomorphism semigroup (and category) of bounded lattices, Pacific J. Math. 35 (1970), 639-647.

6. Z. Hedrlín and A. Pultr, Symmetric relations (undirected graphs) with given semigroup, Monatsh. Math. 69 (1965), 318-322.

7. V. Koubek, Towards minimal binding varieties of lattices, Canad. J. Math. (to appear).

8. V. Koubek and J. Sichler, Small universal varieties of distributive double p-algebras, Glasgow J. Math. (to appear).

9. R. McKenzie and C. Tsinakis, On recovering a bounded distributive lattice from its endomorphism monoid, Houston J. Math. 7 (1981), 525-529.

10. A. Pultr and V. Trnková, Combinatorial, algebraic and topological representations of groups, semi groups and categories, North-Holland, Amsterdam, 1980.

11. B. M. Schein, Ordered sets, semilattices, distributive lattices and Boolean algebras with homomorphic endomorphism semigroups, Fund. Math. 68 (1970), 31-50.

12. J. Sichler, Nonconstant endomorphisms of lattices, Proc. Amer. Math. Soc. 34 (1972), 67-70.

MFF KU, Malostranské NÁM. 25, Praha 1, CZechoslovakia

Department of Mathematics, University of Manitoba, Winnipeg, Manitoba, CANADA R3T 2N2 